\title{
Muséologies
}

Les cahiers d'études supérieures

muséologies

\section{L'incidence des outils pédagogiques sur la visite des adolescents au musée}

\section{Marie-Ève Champagne}

Volume 5, numéro 2, printemps 2011

URI : https://id.erudit.org/iderudit/1033518ar

DOI : https://doi.org/10.7202/1033518ar

Aller au sommaire du numéro

Éditeur(s)

Association Québécoise de Promotion des Recherches Étudiantes en Muséologie (AQPREM)

ISSN

1718-5181 (imprimé)

1929-7815 (numérique)

Découvrir la revue

Citer cet article

Champagne, M.-È. (2011). L’incidence des outils pédagogiques sur la visite des adolescents au musée. Muséologies, 5(2), 132-161.

https://doi.org/10.7202/1033518ar
Résumé de l'article

L'émergence du visiteur " participant » requiert du musée l'évaluation périodique de ses approches éducatives, de façon plus particulière lorsqu'il s'agit d'adolescents. Cette clientèle bien souvent laissée pour compte a été étudiée par Marie-Ève Champagne. Cet article évalue la performance du modèle du «musée constructiviste » de Ceorge E. Hein en tant que stratégie éducative. L'enquête que l'auteure a menée auprès de 325 jeunes lui permet d'établir que ce modèle peut devenir une approche efficace auprès d'une clientèle en constante transformation. 
Marie-Ève Champagne

L'incidence des outils pédagogiques sur la visite des adolescents au musée

L'émergence du visiteur "participant" requiert du musée l'évaluation périodique de ses approches éducatives, de façon plus particulière lorsqu'il s'agit d'adolescents. Cette clientèle bien souvent laissée pour compte a été étudiée par Marie-Ève Champagne. Cet article évalue la performance du modèle du "musée constructiviste" de George E. Hein en tant que stratégie éducative. L'enquête que l'auteure a menée auprès de 325 jeunes lui permet d'établir que ce modèle peut devenir une approche efficace auprès d'une clientèle en constante transformation.

Marie-Éve Champagne a obtenu un baccalauréat en Beaux-arts de l'Université Concordia, spécialisée d'une majeure en peinture et dessin, ainsi que d'une mineure en histoire de l'art. Titulaire d'une bourse d'études supérieures du Canada CRSH 2007-2008 et d'une bourse de maîtrise en recherche du FQRSC 2008-2009, elle détient un diplôme de maîtrise en muséologie de l'Université de Montréal. Elle a occupé différents postes dans le secteur muséal au niveau des services éducatifs, notamment à titre de médiatrice culturelle. Elle débutera un doctorat en histoire de l'art à l'automne 2011.

marie-eve.champagne.1@umontreal.ca 


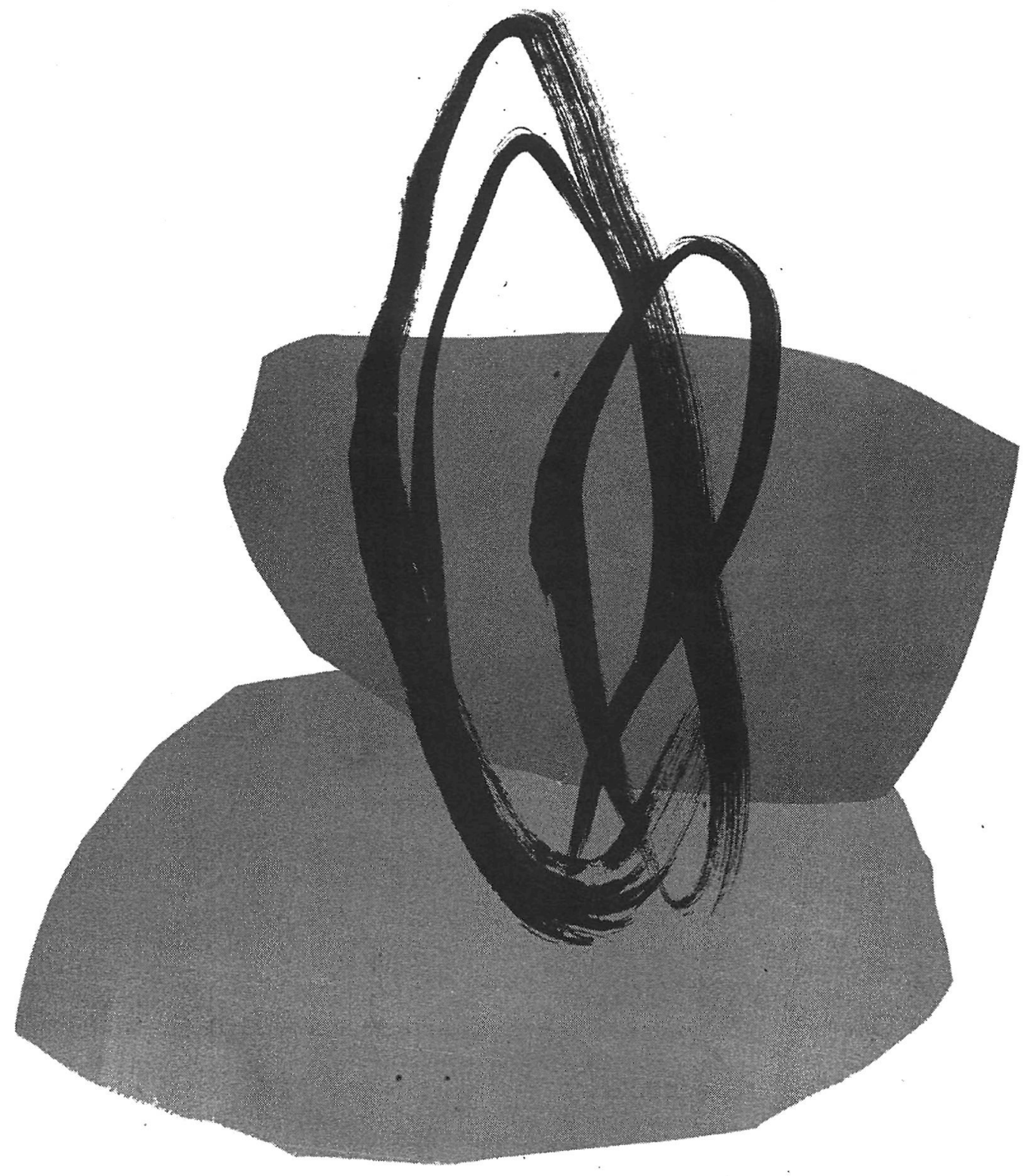


CILMAN, Benjamin I. "Museum Fatigue". Scientific Monthly, vol. 2, $n^{\circ} 1,1916$, p. 62-74.

2

EIDELMAN, Jacqueline, Bernadette COLDSTEIN et Mélanie ROUSTANT. La place des publics: De l'usage des études et recherches par les musées. Paris: La Documentation française, coll. "Musées-Mondes", 2008; et EIDELMAN, Jacqueline. "Qui fréquente les musées à Paris? Une sociographie des publics des musées de France". Publics et Musées, $n^{\circ} 2,1992$, p. 19-47.

3

DUFRESNE-TASSE, Colette et André LEFEBVRE. Psychologie du visiteur de musée: contribution à l'éducation des adultes en milieu muséal. Ville LaSalle: Hurtubise $\mathrm{HMH}$, coll. "Cahiers du Québec: Psychopédagogie», 1996.

4

CAMERON, Duncan, F. "Un point de vue: le musée considéré comme système de communication et les implications de ce système pour les programmes éducatifs muséaux". [1968] Vagues, 1992, p. 271-280; BITCOOD, Stephen. "Évaluation des musées du point de vue de la conception sociale". In. Faire voir, Faire savoir: La muséologie scientifique au présent. SCHIELE, Bernard (dir.). Québec: Musée de la civilisation, 1989, p. 87-106; et JACOBI, Daniel, Anik MEUNIER et Sylvie ROMANO. "La médiation culturelle dans les musées: une forme de régulation sociale". Recherches en Communication, $n^{\circ} 13,2000$, p. 37-60.

5

DIERKINC, Lynn D. "Rôle de l'interaction sociale dans l'expérience muséale". Publics et Musées, $n^{\circ} 5$. 1995, p. 19-43.

6

DUFRESNE-TASSE et LEFEBVRE, op. cit.

7

SOCIÉTE DES MUSÉES QUÉBECOIS. Etude omnibus: Opinion et habitudes de consommation des Québécois dans les institutions muséales du Québec, 2004. <http://www.smq.qc.ca/
Les publications portant sur les visiteurs de musées sont abondantes et exhaustives. Depuis la première étude parue sur le sujet en 1916', de nombreux chercheurs et spécialistes se sont penchés sur cette question. Par leur entremise, nous apprenons à mieux connaître les individus qui circulent dans les expositions d'un point de vue sociologique ${ }^{2}$, les manières dont ils font sens des contenus qui leur sont proposés dans une perspective psychologique ${ }^{3}$ ou encore les rôles sociaux qu'ils jouent dans l'espace communicationnel qu'est le musée 4 . Nous sommes maintenant mieux renseignés sur les manières dont se comporte le visiteur dans une institution muséale et sur les modes d'assimilation ou d'appréciation qu'il met en place. D'une part, le visiteur apprend mieux en interagissant avec les autres ${ }^{5}$ et il apprécie davantage une visite lorsque son imaginaire ou son intellect est sollicité ${ }^{6}$. D'autre part, des travaux menés au Québec ont permis d'en savoir davantage sur les caractéristiques sociodémographiques des visiteurs. Ainsi, une personne est plus susceptible de fréquenter les musées si elle provient d'une région métropolitaine, que son revenu familial est supérieur à la moyenne et qu'elle a un diplôme d'études universitaires'.

Le secteur de l'éducation en milieu muséal est particulièrement lié à l'étude des visiteurs, car l'intérêt porté à ces derniers contribue à l'essor de ce type de recherche ${ }^{8}$. De nombreux travaux dans ce domaine ont été conduits sur les publics adultes et enfants, notamment au Québec, par l'entremise de deux groupes de recherches: le Groupe de recherche sur l'éducation et les musées (GREM) et le Groupe de recherche sur les musées et l'éducation des adultes (GRMEA). A ceux-ci s'ajoutent bien entendu le Groupe d'intérêt spécialisé sur l'éducation et les musées (GISEM), qui constitue à la fois une alliance entre le GREM et le GRMEA, ainsi que d'autres chercheurs canadiens. Pour sa part, le public adolescent demeure le plus méconnu des chercheurs et des institutions muséales.

Considérée comme une phase transitionnelle entre l'enfance et l'âge adulte par les spécialistes ${ }^{9}$, l'adolescence consiste en une période du développement humain 
marquée par le changement. En raison de cela, il est difficile de mesurer précisément les caractéristiques de ce cinquième "âge " de $l^{\prime}$ Homme ${ }^{10}$, ce passage obligé où se développe l'identité et parfois aussi la confusion des rôles. Pourtant, étant donné que la population de ce groupe d'âge est en pleine croissance dans de nombreux pays et qu'elle constitue un bassin important de visiteurs potentiels, il est nécessaire de poursuivre les efforts de recherche sur ce public, afin de maximiser sa présence au sein des musées. Le pouvoir d'influence qu'a la génération adolescente sur la forme que prendront les musées dans l'avenir est considérable.

Teens, if we allow them, have the potential to provide our museums with the fresh perspective and energy required by each new generation as it reinvents and finds the significance of its own cultural patrimony. Museum directors, educators, and marketing experts now recognize increasingly that this audience represents the pulse of contemporary culture. Their thinking will be at the forefront of society within the decade" .

Il est crucial de mieux connaître le public adolescent afin d'atteindre une visée primordiale du musée, soit d'être en phase avec la société dans laquelle il évolue ${ }^{12}$.

L'accord du musée avec le public adolescent passe par une meilleure connaissance des pratiques muséales de ce dernier. L'étude des outils pédagogiques incitant les adolescents à fréquenter le musée constitue un pas dans cette direction. Quels outils incitent davantage les adolescents à visiter un musée: des visites guidées, des ateliers créatifs, des rencontres avec des artistes ou des médiateurs, des films, des concours, des bornes interactives, des démonstrations, des reconstitutions, ou encore des sites Internet dédiés à leur groupe d'âge?

Avant de poursuivre notre réflexion, tentons d'abord d'offrir une définition de ce que nous entendons par "outil pédagogique ", dont nous venons d'énumérer quelques exemples. Alors que le musée est souvent perçu lui-même comme un outil pédagogique ${ }^{13}$, dans la mesure où il constitue une forme de support à l'apprentissage formel dont les écoles peuvent se servir afin de mieux approfondir les contenus abordés en classe, nous préférons, dans
pdf/2004-09-23-LegerMarketing. pdf> (consulté le 23 février 2010). 8 HEIN, George, E. Learning in the Museum. Londres et New York: Routledge, 1998.

\section{9}

HODKINSON, Paul et Wolfgang DEICKE. Youth Cultures. Scenes, Subcultures and Tribes. Londres et New York: Routledge, 2007. 10

ERIKSON, Erik H. Childhood and Society. New York et Londres: W.W. Norton G Company, 1964. 11

SCHWARTZ, Deborah F. "Dude, Where's My Museum? Inviting Teens to Transform Museums". Museum News, septembre-octobre 2005. <http://www.aamus.org/pubs/ mn/MN_SOO5_teenagers.cfm> (consulté le 23 janvier 2010). 12 BERGERON, Yves et LUC DUPONT. Musées et muséologie: nouvelles frontières. Essais sur les tendances. Québec: Musée de la civilisation, 2005. 13

A ce propos, voir notamment BUFFET, Françoise (dir.). Entre école et musée : le partenariat culturel de l'éducation. Lyon: Presses universitaires de Lyon, 1999; et MICHELET SAAS, Marie-Romaine. "Le musée, un outil pédagogique", L'école, n³9, juin 2005. <tp://ftp.geneve.ch/dip/ ecole39/musee_26.pdf $>$ (consulté le 10 avril 2011). 


\section{4}

MEUNIER, Anik. "Les outils pédagogiques dans les musées: pour qui, pour quoi ?". La Lettre de I'OCIM, n 133 , janvier-février 2011, p. 5. <http://ocim.revues.org/648> (consulté le 9 avril 2011).

15

Id., p. 7.

16

FALK, John H. et Lynn D. DIERKING. The Museum Experience. Washington (DC): Whalesback Books, 1992.

17

MEUNIER, op. cit., p. 11. cet article, circonscrire la définition de cette expression au cadre muséal et nous en tenir plutôt à l'explication que propose Anik Meunier, professeure-chercheure aux départements d'éducation et de muséologie de l'Université du Québec à Montréal, dans Les outils pédagogiques dans les musées: pour qui, pour quoi?:

Un outil pédagogique représente un instrument qui privilégie une stratégie ou une manière de procéder dans le but spécifique de soutenir les visiteurs dans l'appropriation des contenus du musée ou de l'exposition ${ }^{14}$.

Toujours selon cette chercheure, l'outil pédagogique renvoie non seulement à la question de "l'apprentissage, mais aussi [au] processus d'acculturation lui-même en contexte d'éducation non formelle ${ }^{\prime 5}$. Influencé par les trois contextes fondamentaux de l'expérience muséale proposés par John H. Dierking et Lynn D. Falk dans l'ouvrage The Museum Experience, soit les contextes personnel, social et physi$q^{q u e}{ }^{16}$, il doit réciproquement en tenir compte et "ne peut se contenter de reproduire un modèle standard " $"$. De cette manière, les formes tangibles de l'outil pédagogique ou "outil éducatif", si l'on traduit mot pour mot la terminologie américaine à ce sujet (educational tool), sont multiples et variées. Elles changent au rythme des interventions et des situations d'apprentissages. Dans cet article, nous ne limitons pas la définition de l'outil pédagogique aux seuls équipements didactiques de nature matérielle. Nous élargissons le spectre de la définition en incluant également les interventions humaines telles qu'une visite guidée ou la rencontre-conférence avec un artiste. Dans cette perspective, l'intervention humaine peut constituer un outil en soi et non seulement un support à la présentation d'un outil. En conséquence, dans le cadre qui nous occupe, le cartel, la borne multimédia, l'enregistrement sonore, la visite guidée, l'atelier pratique, le geocaching et la rencontre avec un expert sont autant de formes d'outils pédagogiques. Si nous revenons sur la définition première de Meunier à laquelle nous adhérons, nous précisons que l'outil pédagogique peut également constituer, à l'instar d'un "instrument" matériel, une intervention humaine. 
Alors qu'au Québec seulement $28 \%$ de la clientèle scolaire pour l'année 2009 était constituée d'élèves du secondaire en comparaison à plus de $64 \%$ d'élèves du primaire ${ }^{18}$, mieux connaître les outils pédagogiques qui incitent les adolescents à prendre part à l'espace muséal constitue une entreprise cruciale. Ultimement, ces informations en main, les institutions pourront mieux orienter leurs approches à partir des intérêts des adolescents et ainsi créer davantage de liens avec ce public particulier.

George E. Hein, chercheur et professeur spécialisé dans le domaine de l'éducation muséale, soutient qu'il y a trois préoccupations nécessaires à l'élaboration d'une théorie de l'éducation muséale: une théorie de la connaissance, une théorie de l'apprentissage et une théorie de l'enseignement, qui se trouve à être une mise en pratique des deux premières' $^{19}$. Dans le contexte muséal, l'auteur marque par ailleurs une préférence pour une théorie de l'éducation particulière, soit le constructivisme. Cette dernière est issue d'une conception de la connaissance présupposant que l'élève construit de nouvelles connaissances à partir de celles qu'il possède déjà, ainsi que d'une conception de l'apprentissage qui suggère que l'élève apprend d'abord par le biais de l'expérience.

Constructivists, with their concern with the schemas and ideas that are already in learner's minds, will be more likely to ask whether the environment is one with which the learner can make any connections. Is there a familiar reference, object, idea or activity that will allow the learner to engage with the issue ${ }^{20}$ ?

Dans son diagramme des théories de l'éducation (voir illustration), Hein met en relation différentes théories selon leur position épistémologique (leur croyance au sujet de la nature de la connaissance) et la psychologie de l'apprentissage qu'elles mettent de l'avant (comment la connaissance se construit-elle?). ${ }^{21}$.
18

INSTITUT DE LA STATISTIQUE DU

QUEBEC. Observatoire de la culture et des communications du Québec. "Tableau 3.5-Visiteurs dans les institutions muséales répondantes selon le type d'institution et la discipline, Québec, 2009", février 2010. <http://www.stat.gouv.qc.ca/ donstat/societe/culture_comnc/ musees/visit_inst_mus_rep_typ_ dis_2009.htm> (consulté le 29 mars 2010).

19

HEIN, Learning in the Museum, op. cit. 20

Id., p. 38.

21

HEIN, George, E. «The Constructivist Museum ». In. HOOPER-GREENHILL, Eilean (dir.). The Educational Role of the Museum. Londres et New York: Routledge, 1999, p. 73-74. 
22

HEIN, George, E. "Education Theories". In. HEIN, Learning in the Museum, op. cit., p. 25.



Ce diagramme met en évidence les distinctions fondamentales entre le constructivisme et d'autres théories de l'éducation bien connues, dont le behaviorisme, qui correspond au cadran inférieur gauche (Stimulis-Réponse).

En transposant le modèle constructiviste issu des sciences de l'éducation au cadre muséal, il est intéressant de se pencher sur l'incidence des outils pédagogiques sur la fréquentation des musées par les adolescents. Avant de rendre compte des résultats d'une enquête que nous avons conduite directement auprès d'adolescents à ce sujet, il importe d'abord de mieux comprendre les différents enjeux de cette problématique.

\section{L'importance du visiteur en éducation muséale}

Selon le Conseil international des musées (ICOM), l'éducation constitue un des trois objectifs fondamentaux au cœur de la mission du musée, aux côtés de la "délectation" et de l'uétude "23. Hein ne minimise pas l'importance de la 
fonction d'éducation dans l'histoire du musée, alors qu'il date ses origines aux premiers musées publics du XVIIIe siècle et qu'il croit qu'elle est aujourd'hui d'une importance cruciale pour les institutions muséales.

For a variety of reasons, ranging from changing definitions of learning and pressures on museums to justify their existence to expending socio-political roles of museums in increasingly self-conscious societies, museum education is increasing in significance. What is learned in museums and how learning takes place is more than a matter of intellectual curiosity. Learning in the museum and understanding visitors' learning has become a matter of survival for museums ${ }^{24}$.

Les propos de Hein renvoient ici non seulement à l'importance de l'éducation à titre de fonction muséale, mais aussi à un acteur essentiel qui sous-tend cette importance: le visiteur.

Le rôle prépondérant du visiteur est mis en évidence dans de nombreuses recherches issues du CISEM. Dans l'ouvrage L'éducation muséale vue du Canada, des États-Unis et de l'Europe: Recherche sur les programmes et les expositions ${ }^{25}$, Michel Allard, Anik Landry et Claire Meunier tentent de classifier l'ensemble des efforts de recherche entrepris concernant les programmes éducatifs muséaux. Ils y présentent les "lignes de force" de la recherche en éducation muséale. Les concepts fondamentaux recensés, soit le sujet (visiteur), l'objet (thématique) et l'agent (guide-interprète ou intervenant), trouvent écho dans d'autres recherches sur la scène internationale ${ }^{26}$. En présentant deux modèles communicationnels dans le secteur de l'éducation muséale, un basé sur la transmission et un second sur une vue plus "culturelle", Eilean Hooper-Greenhill, chercheure à l'Université de Leicester en Angleterre, rappelle les liens qui unissent le domaine des communications à celui de l'éducation muséale ${ }^{27}$. Les modèles offerts par la chercheure présentent sensiblement les mêmes éléments que celui d'Allard, Landry et Meunier, seulement ils sont appelés différemment, soit le "communicateur", le "message-médium" ou le "receveur" ${ }^{28}$. Avec ses modèles, Hooper-Greenhill souligne elle aussi la place fondamentale du visiteur au sein de l'éducation muséale, nonobstant les changements qui l'amènent à passer d'un rôle passif à une participation plus
23

CONSEIL INTERNATIONAL DES MUSEEES. Statuts de l'ICOM : Article 3-Définition des termes. <http:// icom.museum/statutes_fr.html\#2> (consulté le 15 février 2010).

24

HEIN, Learning in the Museum, op. cit., p. 12.

25

EMOND, Anne-Marie. L'éducation muséale vue du Canada, des EtatsUnis et d'Europe: recherche sur les programmes et les expositions. Québec: Editions MultiMondes, 2006. 26

ALLARD, Michel, Anik LANDRY et Claire MEUNIER. "Où va l'éducation muséale? ". In. ÉMOND, L'éducation muséale vue du Canada, des Etats-Unis et d'Europe..., id., p. 9-20.

27 HOOPER-GREENHILL, The Educational Role of the Museum. op. cit., p. 69-70.

28

Id. Termes tirés du modèle de transmission. 
29

Id., p. 67.

30

POULOT, Dominique. Musée et muséologie. Paris: La Découverte, coll. "Repères», 2005, p. 54. 31 DEWEY, John, Albert C. BARNES, Laurence BUERMEYER, Thomas MUNRO, PaUI GUILLAUME, Mary MULLEN et Violette DE MAZIA. Art and Education. Merion (PA): The Barnes Foundation Press, 1929; DEWEY, John. Art as Experience. New York: Perigee, 1934.

32

VAIL COLEMAN, Laurence. The Museum in America: A Critical Study. Washington (DC): American Association of Museums, 1939 , 3 vols.

33

HOOPER-GREENHILL, The Educational Role of the Museum, op. cit., p. 7 . active $^{29}$. A propos de la scène américaine, Dominique Poulot rapporte que le public a joué un rôle déterminant très tôt dans l'histoire des établissements muséaux américains. A son avis, deux influences majeures ont contribué à cet essor et ont considérablement altéré le rôle du visiteur ${ }^{30}$ : d'une part, les écrits du philosophe John Dewey notamment spécialisé en pédagogie, Art and Education et Art as Experience $^{31}$, et, d'autre part, la valorisation de la fréquentation à titre d'indicateur du succès d'une institution muséale mise de l'avant par l'Association des musées américains (AAM) dès $1939^{32}$.

En somme, parmi les trois concepts fondamentaux articulant la question de l'éducation muséale, la prépondérance du public se constate dans un large lot des écrits produits sur le sujet. En effet, comment outrepasser le visiteur dans l'analyse de la fonction d'éducation du musée? N'est-il pas le destinataire de toute entreprise éducative? Par ailleurs, n'est-ce pas d'abord par lui que nous tentons de mesurer les caractéristiques de cette fonction muséale?

\section{Le musée constructiviste}

La fonction éducative d'un musée n'est pas étrangère aux courants issus du domaine de l'éducation. Bien souvent, la manière dont on présente un contenu éducatif est liée à une conception de ce qu'est l'éducation. En regard du cadre muséal qui nous occupe, il semble qu'une préoccupation majeure ressorte, peu importe la théorie de l'éducation sur laquelle se fonde le programme éducatif :

Gradually during the last half-century, and gathering pace in the last 20 years, we can identify a move in education and communication theory towards acknowledging people as active in making sense of their social environments, towards acknowledging both that plural views exist and that they are all legitimate ${ }^{33}$.

Le changement de paradigme dont parle Hooper-Greenhill apparaît particulièrement évident dans les théories constructivistes de l'éducation.

La théorie constructiviste de l'apprentissage s'inspire du développement neurologique. "New and higher-level neural 
structures have to connect to or grow from structures (knowledge) already there " ${ }^{34}$. Dans son ouvrage We're Born to Learn, où elle définit le constructivisme, Rita Smilkstein rappelle un exemple utilisé par le père fondateur de cette théorie, Jean Piaget (1973), et plus récemment par Gopnik et ses collègues (1999): un bébé ne part jamais de rien, il construit ses connaissances à partir de ce qu'il a déjà vécu. Par exemple, c'est à partir de son réflexe inné de sucer qu'il peut accéder à d'autres connaissances, qu'il modifie son comportement selon les situations ${ }^{35}$.

Les origines et les ramifications du constructivisme sont variées. Dans Constructivism in Education, Eric Bredo ainsi que Kenneth $\mathrm{R}$. Howe et Jason Berv ${ }^{36}$ se sont penchés sur le constructivisme considéré d'un point de vue philosophique. Ils ont établi que la pensée constructiviste remonte à Kant, alors qu'il tentait de synthétiser l'empirisme et le rationalisme. Plus récemment, les travaux de Jean Piaget et de Leg Vygotsky, élaborés au cours de la première moitié $\mathrm{du} \mathrm{XX}^{\mathrm{e}}$ siècle dans le secteur de la psychologie cognitive du développement, ont fait d'eux de véritables pionniers du constructivisme tel que nous le connaissons aujourd'hui et tel que le résument Howe et Brev:

Constructivist learning theory has two basic premises: (1) learning takes as its starting point the knowledge, attitudes, and interests students bring to the learning situation, and (2) learning results from the interaction between these characteristics and experience in such a way that learners construct their own understanding, from the inside, as it were ${ }^{37}$.

Poussé à l'extrême, le constructivisme radical met à l'épreuve les limites conceptuelles de cette théorie de l'éducation. Le risque associé à ce type de radicalisme est notamment relevé dans Constructing Constructivism, Epistemological and Pedagogical ${ }^{38}$. Ultimement, il peut mener à une relativisation absolue qui limite la structure sociale de l'apprentissage. Par exemple, quelle est la valeur de l'enseignement magistral si une connaissance n'est validée qu'à partir de soi?

An epistemological stance that leaves knowledge ultimately stranded on private constructions encourages teachers to become overly cautious about what they can confidently claim to know. This, in turn, encourages them to be overly timid about challenging students' personal constructions of the world, for fear of imposing their own personal view of things ${ }^{39}$.
34

SMILKSTEIN, Rita. We're BornTo Learn. Thousand Oaks: Corwin Press, 2003, p. 62.

35

PIACET, Jean. The Child and Reality; Problems of genetic Psychology. New York: Grossman Publishers, 1973; et GOPNIK, Alison, Andrew N. MELTZOFF et Patricia Katherine KUHL. The Scientist in the Crib: Minds, Brains and How Children Learn. New York: Harper Collins, 1999. Cités par SMILKSTEIN, We're Born To Learn, op. cit., p. G1.

36 BREDO, Eric. "Reconsidering Social Constructivism: The Relevance of C.H. Mead's Interactionism". In. PHILLIPS, Dennis (dir.).

Constructivism in Education. National

Society for the Study of Education Yearbook. Chicago: University of Chicago Press, 2000, p. 128; et BERV, Jason et Kenneth R. HOWE. "Constructing Constructivism, Epistemological and Pedagogical». In. PHILLIPS, id., p. 20-21.

37

Id., p. 30-31.

38

Id., p. 38 .

39

Id., p. 38. 
40

CLARK, Richard E. "How Much and What Type of Cuidance Is Optimal for Learning from Instruction? ". In. TOBIAS, Sigmund et Thomas $M$. DUFFY (dir.). Constructivist Instruction: Success or Failure? Londres et NewYork: Routledge, 2009, p. 158.

41

GREENE, Maxine. "Chapter 7: A Constructivist Perspective on Teaching and Learning in the Arts". In. TWOMEY FOSNOT, Catherine (dir.). Constructivism: Theory, Perspectives and Practice. New York: Teachers College Press, 2005, p. 110-131.
Comme le souligne Richard E. Clark dans un chapitre de l'ouvrage Constructivist Instruction: Success or Failure? ${ }^{40}$, il peut être difficile de trouver le niveau de support pédagogique adéquat provenant de l'enseignant à partir des méthodes d'apprentissages constructivistes. En effet, le rôle du professeur dans le processus d'apprentissage doit être redéfini. Quel rapport l'enseignant doit-il entretenir avec la passation des connaissances, si l'acquisition ne s'effectue qu'à partir des expériences personnelles de l'élève? Est-il un intermédiaire superflu qui vient troubler la "construction" de nouvelles connaissances ou bien constitue-t-il avant tout un instigateur de questionnements qui mèneront à la création de nouvelles connaissances liées aux préalables personnels de l'élève?

Dans le texte $A$ Constructivist Perspective on Teaching and Learning in the Arts, Maxine Greene a analysé en détail le constructivisme appliqué dans le secteur des arts' ${ }^{41}$. Ses observations sont avant tout liées à la notion d'interprétation propre à ce contexte particulier, mais elles offrent néanmoins de grandes résonnances avec la question du niveau adéquat de support pédagogique trouvée dans les situations d'apprentissages constructivistes. D'une part, c'est par le désir de découvrir un autre monde que le sien que le spectateur (l'élève) sera amené à vouloir connaître une œuvre. Greene écrit que le professeur doit d'abord sonder sa propre compréhension pour arriver à créer une "conversation " qui amènera un élève à s'approprier une œuvre d'art dans la mesure où elle fera désormais partie de son expérience. Nous pouvons certainement dresser des parallèles entre le rôle de l'enseignant en milieu scolaire et celui du support pédagogique humain dans la structure muséale, qu'il s'appelle un guide, un éducateur ou encore un médiateur. Cela signifie que c'est en traitant son propre savoir dans une optique constructiviste que l'intervenant muséal (guide, éducateur ou médiateur) arrivera à favoriser la création de connaissances chez le visiteur.

Dans une optique muséale, le constructivisme est particulièrement sensible au visiteur : ses intérêts, ses pratiques et l'exercice de son libre-arbitre sont au cœur des 
préoccupations du musée, afin de lui offrir une multitude d'occasions de tisser des liens entre ses acquis personnels et les connaissances liées au contenu d'une exposition, par exemple. Au sujet de sa préférence pour le modèle constructiviste, George E. Hein écrit notamment:

[F]ocusing on visitors, the meanings they attribute to their experiences, and their understandings, is the most useful way to develop exhibitions and programs that will allow visitors to have satisfying museum experiences and allow museums to maximize the inherent potential of objects to contribute to human growth and learning ${ }^{42}$.

Dans cette perspective, le "musée constructiviste", tel que le conçoit Hein, est un modèle qui prévaut de plus en plus dans le monde des institutions muséales. Puisqu'il met d'abord l'accent sur le visiteur plutôt que sur le contenu d'une exposition, contribuant à remplacer les "certitudes du modernisme" par la "fluidité du postmodernisme", le musée constructiviste s'avère très "postmoderne" dans le sens entendu par Eilean Hooper-Greenhill ${ }^{43}$.

L'application concrète du constructivisme au monde des musées est complexe. Variant selon les individus qui "construisent" l'expérience muséale, il demeure néanmoins que certains points ressortent. En opposition au musée de type "systématique", les caractéristiques du "musée constructiviste" sont les suivantes: des expositions où la circulation est plus libre, où l'on emploie des méthodes d'apprentissage multiples et variées, et où prévalent des possibilités pour le visiteur de créer des connexions avec des concepts familiers ${ }^{44}$. De cette manière, plus les musées connaîtront les adolescents et les outils pédagogiques qui les stimulent, plus ils seront à même de créer des occasions de rencontres valorisantes et valorisées auprès de ce public particulier.

\section{La présence des adolescents au musée}

La recherche scientifique a longtemps apposé des étiquettes aux adolescents. Jusqu'aux années 1980, ce groupe de jeunes a surtout fait l'objet d'études menées dans la perspective sociologique d'une sous-culture marginalisée et inscrite dans la rébellion ou la délinquance ${ }^{45}$. Au-delà de
42

HEIN, Learning in the Museum, op. cit., p. 12-13.

43

[Traduction libre] HOOPERGREENHIL, The Educational Role of the Museum, op. cit., p. 7 .

44

HEIN, "The Constructivist

Museum», op. cit., p. 77 .

45

HODKINSON, Paul. "Youth Cultures: A Critical Outline of Key Debates ". In. HODKINSON, Paul et Wolfgang DEICKE (dir.). Youth Cultures. Scenes, Subcultures and Tribes. Londres et New York: Routledge, 2007, p. 8. 
46

BANKS BEANE, Deanna.

"Museums and Healthy Adolescent

Development: What We Are

Learning from Research and

Practicen. Journal of Museum

Education: Adolescence: Growing Up

in Museums, vol. 25, n³,2000, p. 4.

47

Ibid.

48

Tamara LEMERISE ("Les adolescents au musée: enfin des chiffres!". Publics et musées. Lyon: Presses universitaires de Lyon, n 15,1999 , p. 10) souligne que les adolescents (12-17 ans) sont mal compris dans la grande catégorie des $15-24$ ans normalement utilisée pour classifier ce groupe d'âge.

49

LEMERISE, id., p. 10.

50

LEMERISE, Tamara et Dany

LUSSIER-DESROCHERS. "La relation musées-adolescents démystifiée". In. LEMERISE, Tamara, Dany LUSSIER-DESROCHERS et Vitor MATIAS (dir.). Courants contemporains de recherche en éducation muséale. Montréal : Éditions MultiMondes, 2002, p. 49-64.

51

1d., p. 49.

52

Id., p. 50. la disparité des définitions de l'adolescence trouvées dans les propos des spécialistes, un consensus général détermine que l'adolescence "begins with pubescence and the development of significant hormone-induced physical changes, several of which are major milestones for adolescents-and their parents and teachers " ${ }^{46}$. Sur les plans affectif et psychologique, en général, plusieurs changements accompagnent cette période de grandes transformations hormonales: "fear, worry, feelings of inferiority and perhaps moodiness are among the more common indications of the impact of an adolescent's changing body on his emotional state ${ }^{47}$. En ce qui concerne les tranches d'âges précises de l'adolescence, certaines études situent le début de l'adolescence à 10 ans, tandis que d'autres le positionnent plutôt entre 12 ans et 17 ans.

Puisque les données sur les adolescents sont limitées, notamment en raison des catégories de classement des visiteurs par âge ne permettant pas le calcul précis de la fréquentation des adolescents au musée ${ }^{48}$, il est difficile de déterminer précisément le nombre de visiteurs adolescents dans les institutions muséales. Encore aujourd'hui, malgré l'appel lancé par Tamara Lemerise en 1999 dans son article intitulé "Les adolescents au musée : enfin des chiffres", il demeure ardu de se procurer des statistiques spécifiques sur les adolescents ${ }^{49}$.

Dans un article publié dans l'ouvrage Courants contemporains de recherche en éducation muséale ${ }^{50}$, Tamara Lemerise et Dany Lussier-Desrochers, s'appuyant sur une étude menée auprès de 2400 élèves du secondaire, proposent de démystifier la relation qu'entretiennent les musées et les adolescents. Ces chercheurs constatent notamment que, tandis que les collaborations entre les milieux scolaires de niveau primaire et les musées sont nombreuses et diversifiées, il en va autrement pour les écoles secondaires et les adolescents $^{51}$. Ils expliquent cette disparité par le jeu de deux facteurs : 1) les structures organisationnelles des établissements scolaires secondaires qui ne maximisent par les collaborations avec le musée et 2) la perception singulière que les adolescents entretiennent à propos du musée ${ }^{52}$. Les auteurs démontrent par ailleurs que la majorité des 
adolescents perçoivent positivement le musée, mais qu'ils jugent que ces établissements s'adressent aux adultes plutôt qu'à eux. Ainsi, il semble que les musées ne soient pas considérés inutiles par les adolescents qui leur « reconnaissent une fonction sociale, éducative et économique " ${ }^{53}$, mais qu'ils sont plutôt mal adaptés à leurs besoins en ne permettant que de "marcher, de lire et de regarder $"{ }^{54}$.

La disparité entre la perception positive des musées par les adolescents et leur fréquentation moins importante est particulièrement évocatrice du manque de correspondance entre l'offre culturelle des musées et les besoins particuliers des adolescents. Selon une autre étude priorisant cette fois la perspective des musées au sujet des partenariats existants entre le musée, l'école secondaire et les adolescents, Tamara Lemerise et Brenda Soucy concluent que, somme toute, le musée s'avère intéressé et désireux de desservir adéquatement le public adolescent ${ }^{55}$. Ces propos rejoignent ceux de Deanna Banks Beane, qui estime qu'on observe depuis les années 1990 un intérêt croissant envers la création de liens entre les musées et les adolescents ${ }^{56}$. Au Québec, nous n'avons qu'à penser aux publicités récentes du Centre des sciences de Montréal, destinées explicitement aux adolescents. Les acteurs, le traitement visuel et le rythme sont spécifiquement ciblés pour ce groupe. Sur la scène américaine, la création du Teen Museum à Chicago est un exemple évident de cet effort de s'ouvrir aux adolescents. L'intérêt croissant des musées à l'égard des jeunes s'est cependant développé inégalement selon les institutions. Des statistiques au sujet de la fréquentation muséale permettent d'établir que seulement $28 \%$ de la clientèle scolaire québécoise pour l'année 2009 était constituée d'élèves du secondaire comparativement à plus de $64 \%$ d'élèves du primaire ${ }^{57}$. Ces chiffres rappellent la situation telle qu'elle doit être vue : bien que le musée soit perçu idéologiquement de manière positive par les adolescents et qu'il soit disposé à desservir convenablement ce public, il importe de concrétiser ces observations par une fréquentation assidue. Les musées doivent multiplier leurs efforts pour intéresser les adolescents, et pas seulement les adultes ou les enfants.
53

Id., p. 58.

54

Id., p. 59.

55

LEMERISE, Tamara et Brenda SOUCY. "Le partenariat entre les musées, les adolescents et les écoles secondaires du Québec: le point de vue des musées". Revue canadienne de l'éducation, vol. 23, n $1,1998, p .12$. 56

BANKS BEANE, op. cit., p. 3 .

57

Pourcentage calculé à partir des chiffres distribués par l'Institut de la statistique du Québec. Voir: INSTITUT DE LA STATISTIQUE DU QUÉBEC. "Tableau 4 - Visiteurs dans les institutions muséales répondantes selon le type d'institution et la discipline, Québec, 2009". <http:// www.stat.gouv.qc.ca/donstat/ societe/culture_comnc/musees/ tab_synthese/tusynthese_og.htm> (consulté le 22 juin 2010). 



Vue des écoles ayant participé à l'étude : Martin Luther King Jr Middle School (image du haut) et El Camino High School (image du bas), Oceanside, CA. Photos: Marie-Eve Champagne, 2011. 




Vue de geocachers à l'œuvre

Photo: Marie-Eve Champagne, 2011. 
58

LEMERISE, op. cit., p. 9 .

59

Centre des sciences de Montréal.

Exposition permanente.

60

Exposition présentée à la Cité des sciences et de l'industrie (CSI) du 21 octobre 2008 au 3 janvier 2010. 61

SCHIELE, Bernard et Emlyn $\mathrm{H}$. KOSTER. La Révolution de la muséologie des sciences: vers les musées du $\left.X X\right|^{e}$ siècle? Lyon: Presses universitaires de Lyon, 1998, p. 189.

\section{Les outils pédagogiques destinés aux adolescents}

Vers la fin des années 1990, une réorientation "socio-éducative" des missions éducatives des musées - où les questions d'ordre communautaire et humain sont davantage prises en compte - a notamment suscité un intérêt croissant des musées envers les adolescents ${ }^{58}$. Depuis, dépendant des institutions, l'état de la situation a beaucoup changé ou est demeuré le même. Par exemple, lors d'une visite dans un musée de sciences, il est généralement fréquent de rencontrer des bornes interactives ou des stations multimédias disposées à l'intérieur des expositions. Conçues bien souvent tant pour les enfants que les adolescents, elles sont particulièrement attirantes pour l'œil adolescent. Au Québec, l'exposition Imagine! 59 présentée au Centre des sciences de Montréal (CSM) est un exemple patent de l'emploi abondant de ce type d'outils pédagogiques. Par ailleurs, on remarque la mise sur pied de jeux multimédias coopératifs conçus pour les publics enfants et adolescents, comme cela a été le cas au CSM avec Mission Caïa, Snowbirds et Mars 2030, ainsi qu'à la Cité des sciences et de l'industrie de Paris avec le jeu de simulation tiré de l'exposition Épidémik l'exposition "contagieuse "60. Ces jeux sont parfois animés par des intervenants, parfois par un programme informatique. Cénéralement, ils requièrent une participation active des visiteurs où ces derniers influencent le déroulement de l'action à l'aide d'une plateforme technologique.

Si l'on met en relation ce type d'outils avec les différents modèles de transmission des connaissances du musée, ce genre d'approche se classe dans la troisième et dernière tradition des modèles cognitifs décrits par Bernard Schiele et Emlyn H. Koster :

La troisième tradition plus récente correspond à ce qu'il est convenu d'appeler une approche constructiviste. Elle suit les besoins spontanés et les intérêts du visiteur et prend appui sur ses activités. Pour cela, elle essaie de favoriser sa libre expression, sa créativité et son savoir-être. Elle met en avant une découverte autonome par le visiteur et l'importance des tâtonnements dans un processus de construction du savoir ${ }^{6}$. 
L'accent mis sur le public adolescent par les musées de sciences est moins visible dans les autres types d'institutions. Aussi est-il plus rare de voir des outils pédagogiques spécifiquement destinés aux jeunes dans les musées d'art, par exemple. Ce sont souvent des programmes éducatifs de longue durée impliquant le milieu communautaire qui sont mis en place, comme ceux que l'on trouve au Musée des beaux-arts de Montréal (Musée en Partage), au Musée d'art contemporain de Montréal (Arrimage) ou encore dans les maisons de la culture par le biais du Programme de médiation culturelle de la ville. Bref, les outils pédagogiques destinés spécifiquement aux adolescents sont peu nombreux. Ils ciblent souvent à la fois les enfants et les adolescents, voire élargissent leur portée jusqu'aux jeunes adultes ${ }^{62}$. Dans cette perspective, se concentrer sur l'étude des outils pédagogiques incitant les adolescents à visiter les musées semble une initiative prometteuse pour ceux qui souhaitent développer une offre éducative à l'intention de ce public.

\section{De la théorie à la pratique - faits saillants de notre enquête}

En raison du manque de publications directement en lien avec le public adolescent, nous avons entrepris de sonder un groupe de jeunes au sujet de leurs usages et de leurs préférences en matière d'outils pédagogiques. Cette initiative vise à découvrir dans quelle mesure certains d'entre eux ont un potentiel intéressant pour développer efficacement ce public particulier. Nous répondons par ailleurs aux conclusions de Tamara Lemerise et Brenda Soucy ${ }^{63}$, qui recommandent fortement la poursuite d'études de type enquête au sujet des adolescents au musée. Enfin, analysant les résultats obtenus en nous référant au modèle constructiviste, nous tentons de fournir des pistes de réponses aux questions soulevées précédemment.

\section{Méthodologie}

Parmi les différentes options possibles quant à la manière d'acquérir des données primaires sur le point de vue des adolescents, nous avons choisi d'entreprendre une enquête

\section{2}

Par exemple, le guide multimédia du Musée du Louvre, à Paris, est un outil populaire auprès des jeunes et cible les 30 ans et moins plutôt que seulement les adolescents. 63

LEMERISE, Tamara et Brenda SOUCY, "Le point de vue d'adolescents montréalais sur les musées". Revue canadienne de l'éducation, vol. 24 , n० 4, 1999, p. 366. 
64

Cet article s'appuie sur une recherche entreprise dans le cadre d'un travail dirigé qui avait pour sujet d'étude l'incidence des outils éducatifs sur la visite du public adolescent dans les musées d'art contemporain plus spécifiquement. Nous avons choisi ici de présenter seulement les résultats qui s'appliquent aux musées en général. Pour consulter les résultats complets, y compris ceux qui traitent spécifiquement du champ de l'art contemporain, se référer à: CHAMPACNE, Marie-Eve. L'incidence des outils éducatifs sur la visite du public adolescent dans les musées d'art contemporain. Rapport de travail dirigé M.A. (muséologie), Université de Montréal, 2010. par voie de sondage ${ }^{64}$. Nous avons donc administré un questionnaire composé de 25 questions à un échantillon précis : un groupe de 325 étudiants de niveau secondaire de la ville d'Oceanside, dans le comté de San Diego en Californie. Notre questionnaire était constitué de trois sections principales: 1) trois questions portant sur les loisirs et les intérêts, 2) douze questions sur les pratiques muséales et la relation à l'art contemporain et 3) neuf questions démographiques; en plus d'une question sur l'appréciation générale des musées en guise de conclusion. Le questionnaire a été distribué sous forme écrite, principalement constitué d'échelles de Likert, une méthode qui permet de quantifier le degré d'accord ou de désaccord du participant en lien avec une affirmation posée, et dont la terminologie variait en fonction des questions posées et des choix de réponses. Il a été administré dans le cadre de cours d'histoire prodigués dans deux écoles différentes, soit une de niveau high school (HS) et une de niveau middle school (MS). Nous nous sommes associée à deux écoles qui disposaient d'un large bassin de répondants auquel nous avions accès. La première école, Martin Luther King Jr. Middle School, regroupe une population d'élèves inscrits aux niveaux 6 , 7 et 8 . Le second établissement scolaire ayant participé à l'enquête est l'El Camino High School, où sont inscrits des élèves de niveaux $9,10,11$ et 12. Le questionnaire prenait en moyenne dix minutes à compléter. Les données récoltées ont été traitées selon une échelle nominale avant d'être transposées et analysées avec le logiciel Statistical Package for the Social Sciences (SPSS).

\section{Quelques résultats}

L'analyse des différentes réponses obtenues lors notre sondage à l'aide du logiciel SPSS nous a permis de constater plusieurs points intéressants concernant les liens entre les adolescents, les outils pédagogiques et les musées. Avant de présenter les faits saillants de notre étude, soulignons un résultat qui justifie à lui seul le propos de cette recherche: $66,1 \%$ des adolescents maintiennent qu'ils visiteraient plus souvent les musées si ceux-ci leur offraient des outils pédagogiques qui leur conviennent. Dans cette optique, notre 
prémisse de départ, à savoir que les outils pédagogiques ont une incidence sur la fréquentation et les pratiques de visite muséale des jeunes, a été confirmée par ce résultat.

Notons également quelques faits pertinents au sujet de notre échantillon: d'une part, l'âge des répondants se situe entre 13 et 19 ans. Pour des fins d'analyses comparatives, nous avons dès le départ divisé nos répondants en deux catégories, permettant une distinction immédiate entre deux groupes d'âge : 1) les élèves de middle school (MS) et 2) les élèves de high school (HS). En somme, les adolescents de la catégorie MS sont âgés entre 13 et 15 ans, alors que ceux de la catégorie HS ont de 14 à 19 ans. Respectivement, la moyenne d'âge au sein de ces groupes est de 13,5 ans pour le premier et de 16 ans pour le second. D'autre part, en ce qui concerne le sexe des répondants, l'échantillon est composé à $42 \%$ de garçons et $58 \%$ de filles.

\section{Les loisirs et les méthodes d'apprentissage face aux pratiques muséales}

Nous avons commencé notre enquête par l'analyse des pratiques de loisirs des adolescents. Il importait de mieux connaître ces dernières afin de vérifier les manières dont elles peuvent être liées aux outils pédagogiques du musée. Parmi les points constatés, nous avons d'abord remarqué que les musées ont très peu de succès à convaincre le visiteur adolescent que leur offre culturelle est un loisir intéressant. Parmi une liste de treize loisirs à identifier selon le niveau d'importance qui leur est accordé, visiter un musée est celui qui se révèle être le moins important : visiter un musée est un loisir peu ou pas du tout important pour plus de $80 \%$ des jeunes. Bien que ce constat puisse sembler prévisible en raison de l'étiquette "ennuyante" attribuée traditionnellement aux musées par les jeunes ${ }^{65}$, il n'en demeure pas moins préoccupant. Cette situation est inquiétante: en manquant de divertir les jeunes, les musées échouent à l'un de leurs objectifs fondamentaux envers ce public, soit d'exposer entre autres à des fins de plaisir ${ }^{66}$. Il n'est donc pas surprenant que plus de $65,3 \%$ des répondants ne sont

\section{5}

LEMERISE et LUSSIERDESROCHERS, op. cit., p. 60. 66

ICOM. Code de déontologie: Glossaire, Musée. <http://icom.museum/quefaisons-nous/normes-professionnelles/code-de-deontologie/L/2. html?tx_hpoindexbdd_ ethics[uid] $=35 \mathrm{GCH}$ ash=ob2c611563\#sommairecontent> (consulté le 5 janvier 2010). 
67

[Traduction libre] DEAN, David. Museum Exhibition:Theory and Practice. Londres et New York: Routledge, 1996, p. 163

68

Musée du Louvre. Le guide multimédia du musée du Louvre, monguide.louvre. fr. Présentation à la presse, p. 1. <http://www.louvre.fr/media/ repository/ressources/sources/ pdf/src_document_53690_v2_ m56577569831201199.pdf> (consulté le 7 janvier 2011). tout simplement pas allés dans un musée au cours de la dernière année.

Par ailleurs, nos résultats soulèvent un exemple particulier quant au décalage présent entre les intérêts des jeunes et le monde des musées. De fait, alors que la lecture constitue un loisir peu ou pas du tout important pour plus de la moitié des répondants, soit $55 \%$ d'entre eux, nous constatons que les musées font face à un sérieux problème. Sachant que le cartel ou le cartel allongé - ce "graphique textuel donnant de l'information " ${ }^{67}$ - constitue l'outil pédagogique principal de nombreux musées, il est clair que, du point de vue des jeunes, cela ne contribue pas à concevoir le musée comme un lieu de loisir. Bien sûr, pour pallier à cette situation, nous ne proposons pas de bannir toute transmission d'information par la lecture. Cependant, il pourrait être intéressant pour les institutions muséales d'élaborer des outils pédagogiques qui exploitent simultanément la forme écrite et un autre format, comme un support visuel, auditif ou encore tactile. Un exemple intéressant d'une tentative allant dans ce sens est le guide multimédia qui a été mis sur pied par le musée du Louvre, à Paris. En effet, par le biais d'un appareil à écran tactile, ce dernier offre au public une panoplie de modalités de médiation interactives. "La plateforme propose, notamment, les contenus audio, la vidéo, les animations vidéo, le texte, les jeux interactifs et les enregistrements 'toucher pour écouter' " ${ }^{68}$. Tout cela, en plus de la possibilité de sélectionner son profil personnel et d'utiliser l'outil pour aider à se repérer à l'intérieur du musée. Même si le guide s'adresse à un large public et non spécifiquement aux adolescents, il demeure néanmoins que le support employé est très familier aux jeunes et constitue une avenue intéressante pour tenter de transmettre de nouvelles connaissances dans une optique constructiviste. Un autre outil qui emploie moins la lecture, mais qui montre tout de même la créativité qui peut encore être mise de l'avant lorsque vient le temps de développer de nouveaux outils, est le geocaching. En effet, le geocaching constitue un filon que certaines institutions emploient pour étendre leur présence à l'extérieur des murs du musée et rejoindre un public qui fréquente peu ou pas beaucoup 
les institutions muséales, tout en employant des moyens qui sont susceptibles de plaire aux jeunes. Cette activité consiste à rechercher des endroits spécifiques (geocaches) à l'aide d'un système de positionnement mondial (CPS), où se trouvent des items spéciaux à manipuler, à troquer, à emporter chez soi ou encore à transporter dans une autre geocache. Il est aussi possible de rendre compte en ligne de l'histoire entourant la trouvaille de la geocache (sur le site www.geocaching.com), où des comptes-rendus, des images et la liste des personnes ayant trouvé la geocache témoignent des expériences des participants. Cette initiative est en croissance au sein de nombreux musées et centres d'interprétation, en particulier aux États-Unis. Ainsi, les musées désireux de développer de nouveaux publics, surtout celui des jeunes, peuvent se tourner vers l'intégration de cette activité. Les musées possédant des collections extérieures semblent particulièrement favorables à la prise en considération du geocaching. À Montréal, par exemple, une collection comme celle que possède le Musée de Lachine, avec son vaste parc de sculptures publiques, pourrait certainement être propice à la mise en place d'une initiative éducative intégrant cette activité. Ici aussi, séparer le jeu de l'apprentissage peut sembler ardu. Néanmoins, d'un point de vue constructiviste, la forte présence de composants technologiques, tels que le GPS et l'Internet, combinés à l'implication directe de l'activité, sont des éléments intéressants lorsque vient le temps d'élaborer un outil pédagogique destiné aux adolescents. Le défi pour tout musée est d'établir un équilibre qui favorise pleinement la construction de nouvelles connaissances.

Le décalage présent entre les moyens de transmission de contenus fréquemment employés par les musées et les intérêts des adolescents est par ailleurs renforcé par l'analyse des méthodes d'apprentissage préconisées par ces derniers. Nos résultats établissent que, parmi les méthodes d'apprentissage que nous leur avons proposées, les adolescents sont nombreux à considérer que la lecture est peu importante dans la démarche d'apprentissage. Ainsi, alors que $52,6 \%$ des répondants lui accordent peu ou pas du tout d'importance, un décalage entre les pratiques éducatives 
69

LEMERISE et SOUCY, "Le partenariat entre les musées, les adolescents..." op. cit., p. 365.

70

BANKS BEANE, op. cit., p. 4.

71

KEATING, Daniel P. «Adolescent Thinking". In. FELDMAN, S. Shirley et Glen R. ELLIOT (dir.). At the Threshold:The Developing Adolescent. Cambridge (MA) et Londres: Harvard University Press, 1990. p. 54-89. couramment trouvées dans les musées et les méthodes d'apprentissage est observable dans ce cas également.

De tels résultats sont éloquents : la lecture à elle seule se révèle être un moyen de communication et de transmission de connaissances peu recommandable si l'on souhaite attirer les adolescents au musée. Les musées gagneraient certainement à rejoindre les intérêts des adolescents en développant des outils pédagogiques dans une optique constructiviste, exploitant ainsi davantage les moyens pouvant faire le pont avec le vécu des jeunes. Ceux qui sont de toute évidence davantage susceptibles de s'intéresser à des activités articulées autour de la participation directe ${ }^{69}$ seraient plus facilement rejoints par des outils pédagogiques qui reprennent des modes d'apprentissage basés sur l'expérience passée et présente. Par ailleurs, le développement de la pensée abstraite ${ }^{70}$ et du jugement critique ${ }^{71} \mathrm{au}$ cours de l'adolescence accentue la nécessité de mettre sur pied des outils pédagogiques par lesquels les jeunes peuvent exercer davantage ces capacités cognitives.

De plus, une corrélation observée entre le niveau d'importance du loisir visite d'un musée et la méthode d'apprentissage Par la lecture corrobore nos précédents constats. Notre étude révèle effectivement que plus les adolescents aiment apprendre par la lecture, plus ils considèrent la visite d'un musée comme un loisir important. Nous croyons que cette corrélation s'explique notamment par la prédisposition favorable des jeunes à l'endroit des outils pédagogiques déjà mis en place par les musées et s'accordant mieux avec leurs expériences personnelles. En d'autres mots, plus les adolescents sont habitués à construire leurs connaissances par des moyens communs à ceux des musées, plus ils apprécient leur visite au musée et plus ils y accordent d'importance. Ce raisonnement s'appuie également sur une autre corrélation observée selon laquelle plus un adolescent accorde de l'importance au loisir de la lecture, plus il est réceptif à l'ensemble des outils pédagogiques proposés par les musées en général. 


\section{Les pratiques muséales}

Nos résultats concernant les pratiques muséales des jeunes rappellent l'importance des facteurs externes à l'adolescent. L'influence des parents, et plus particulièrement de la mère, est cruciale lorsque nous considérons les pratiques muséales des jeunes. Plus cette dernière est instruite, plus l'adolescent développe une appréciation générale des musées, une impression d'ouverture à leur égard, et la fréquence de ses visites (annuelles) est supérieure. Ces résultats confirment le rôle traditionnel des femmes dans la passation des pratiques culturelles ${ }^{72}$, notamment la fréquentation muséale.

De plus, alors que l'Activité scolaire est l'incitatif contextuel principal pour plus de $56 \%$ des répondants et que près de $55 \%$ d'entre eux disent aller plus souvent au musée avec leur école qu'avec toute autre entité sociale, il ressort que l'environnement scolaire est un facteur non négligeable face aux pratiques muséales des adolescents. Il importe donc pour les musées d'aller au-delà des contraintes et de parer au peu de partenariats qu'ils entretiennent avec les écoles secondaires, surtout en comparaison à ceux plus largement établis avec les établissements primaires ${ }^{73}$.

En ce qui a trait à la perception qu'ont les adolescents du degré d'ouverture des musées à leur égard, nos résultats montrent que les jeunes considèrent en majorité $(60,3 \%)$ que les musées leur sont "ouverts". Nous observons également que l'âge semble influencer positivement cette position, alors que les élèves de high school sont plus nombreux à penser ainsi ( $67,8 \%$ contre $53,1 \%$ pour les plus jeunes). Ce sont donc les jeunes au début de l'adolescence qui semblent le moins concernés par l'offre muséale.

\section{Les outils pédagogiques et leur réception}

En ce qui concerne la réception des outils pédagogiques par les adolescents, nous avons par ailleurs découvert que le sexe et l'âge influencent la prédisposition positive ou négative des jeunes à leur égard. D'une part, les filles sont plus réceptives à l'ensemble des outils pédagogiques, à
72

DONNAT, Olivier. Les pratiques culturelles des Français à l'ère numérique: Eléments de synthèse 1997-2008. 2008. <http://www. pratiquesculturelles.culture.gouv.fr/ doc/08synthese.pdf> (consulté le 12 juin 2010).

73

LEMERISE et LUSSIER-

DESROCHERS, op. cit., p. 50; et LEMERISE et SOUCY, "Le point de vue d'adolescents montréalais sur les musées", op. cit., p. 11. 
l'exception des outils Présentation de films ou documentaires et Discussion avec un éducateur muséal. Quant à la variable catégorie (MS ou HS, qui témoigne indirectement de l'âge des répondants), les élèves de HS sont plus susceptibles d'apprécier l'ensemble des outils pédagogiques proposés que le sont les élèves de MS, à l'exception de l'outil visite virtuelle où 18,2\% d'élèves de MS disent apprécier ou beaucoup apprécier cet outil, contre $17,7 \%$ d'élèves de HS.

Lorsque nous avons porté notre attention sur les corrélations possibles entre les outils pédagogiques et différentes variables, nous avons remarqué que plusieurs dépendances existent, mais demeurent peu significatives. Cependant, quelques-unes d'entre elles semblent plus pertinentes et méritent d'être soulevées. D'une part, comme nous l'avons mentionné plus haut, plus la lecture est un loisir important pour l'adolescent, plus ce dernier a tendance à aimer tous les types d'outils pédagogiques proposés. D'autre part, une corrélation est observée entre l'outil pédagogique Information en ligne et le niveau d'importance des technologies dans le quotidien des adolescents. En effet, il semble y avoir une corrélation positive entre des technologies qui sont sur le même support que cet outil. En d'autres mots, parmi les choix de réponses que nous avions offerts, ce sont les technologies qui sont sur ordinateur, telles que Courriels et clavardage, Webcam, et Internet et blogging, qui ont révélé être liées à l'appréciation de l'outil Information en ligne. Ainsi, cette corrélation appuie notre argumentation selon laquelle l'approche constructiviste consiste en une option à considérer sérieusement dans la conception d'outils à fins éducatives en contexte muséal. Elle démontre clairement que plus un outil est lié aux connaissances et aux expériences déjà acquises par les adolescents, plus il sera apprécié par ces derniers.

Enfin, nous avons déterminé que malgré les différences constatées selon les variables étudiées, les adolescents constituent une population somme toute homogène eu égard à leur relation aux outils pédagogiques. Sur un point en particulier, ils se rejoignent manifestement : leur intérêt pour des outils pédagogiques nécessitant leur 
implication directe. Effectivement, tant chez les élèves de MS que chez ceux de HS, tant chez les filles que chez les garçons, les outils pédagogiques Activité participative et Bornes ou stations interactives sont ceux qui sont les plus appréciés. Sur ces points, nos résultats corroborent ceux de Tamara Lemerise et Brenda Soucy ${ }^{74}$, qui ont également souligné l'importance de la participation active chez les adolescents. Il paraît donc crucial pour tout musée désirant accomplir sa mission auprès des jeunes d'offrir des outils pédagogiques qui mettent de l'avant l'implication directe de ces visiteurs. Visiblement, les cartels et les visites commentées ne suffisent pas.

\section{Conclusion}

La place du visiteur a profondément changé au cours des dernières décennies. D'abord peu priorisé en comparaison aux collections, le visiteur est progressivement passé d'un rôle passif à un rôle beaucoup plus actif 75 . Par ailleurs, l'intérêt croissant des musées à son endroit a conduit à la création de nouveaux outils pédagogiques visant à accrô̂tre la fréquentation et la compréhension du public. Le "musée constructiviste" évoqué par George $\mathrm{E}$. Hein repose sur une vision de l'éducation que l'ensemble des institutions muséales devraient adopter si elles souhaitent véritablement s'inscrire en continuité avec le changement de paradigme vécu par le visiteur dont parle Eilean HooperGreenhill17 ${ }^{6}$, au sein duquel ce dernier est désormais perçu comme un acteur proactif dont l'individualité influence l'expérience muséale et les apprentissages qui s'y rattachent. Se positionnant en faveur d'un apprentissage ayant pour bases les connaissances préexistantes pour en construire de nouvelles, le constructivisme est véritablement tourné vers les intérêts et les expériences du visiteur. De cette manière, il permet d'offrir des outils pédagogiques qui arrivent davantage à susciter une véritable appropriation de l'offre du musée. Considérer les outils pédagogiques selon une approche constructiviste permettrait d'augmenter la fréquentation d'un public particulièrement négligé : les adolescents. Tandis que la nouvelle attention accordée au visiteur ne cesse de s'accroître pour le public en général,
LEMERISE et SOUCY,

"Le partenariat entre les musées, les adolescents...", op. cit., p. 365. 75

HOOPER-CREENHILL, The Educational Role of the Museum, op. cit., p. 67.

76

HOOPER-GREENHILL, The Educational Role of the Museum, op. cit., p. 7 . 
77

HOOPER-CREENHILL, Eilean. Museums and Education: Purpose, Pedagogy, Performance. Londres et New York: Routledge, 2007, p. 1. il semble en effet que cette catégorie particulière de visiteurs soit délaissée.

En conclusion, l'ensemble de notre recherche démontre clairement les bénéfices que peut tirer un musée à orienter le développement de ses outils pédagogiques selon une approche constructiviste, où les expériences et les connaissances des adolescents sont à la base de celles que les outils pédagogiques visent à faire acquérir. C'est en tenant compte de l'expérience et des intérêts des jeunes que les musées arriveront à créer des situations d'apprentissage qui favorisent leur présence au musée. Pour y parvenir, il est primordial de poursuivre les efforts de recherche à l'endroit du public adolescent et de multiplier les occasions d'en apprendre davantage sur des moyens susceptibles de modifier la situation actuelle. Toutefois, même si notre étude présente avant tout un propos articulé autour du rôle du musée dans l'amélioration de la fréquentation et de l'appréciation des musées par les jeunes, il ne faut pas négliger une autre question d'importance qui n'a pas été abordée ici, soit le pouvoir qu'ont les adolescents sur la situation qui nous préoccupe. En rejetant les idées reçues et en démontrant une attitude ouverte, ceux-ci parviendraient à créer une rencontre encore plus fertile avec le musée. Dans ces conditions, peut-être pourrions-nous concrétiser davantage le désir de démocratisation du musée? Peut-être enfin embrasserions-nous pleinement l'optimisme du "post-musée" dont parle Hooper-Greenhilln? 
sur la visite des adolescents au musée 
Summary

Translated by Philippe Lagrange

The Influence of Educational

Tools on Visits by Teenagers

to the Museum 
Over the last few decades the role of the museum visitor has deeply changed. On first analysis, the emphasis was being placed on collections; progressively, the visitor's passive role shifted to become much more participative. This article takes a position on the "constructivist museum" as described by Hein as a paradigm shift that offers educative strategies that we feel every museum institution should adopt. We advocate a form of learning based on the visitor's previous knowledge in order to acquire new learnings. Constructivism applied to museums seeks to develop and deliver educative tools that are likely to lead to a true appropriation of the museum, particularly amongst teenagers.

Given the lack of literature on the subject, we undertook a survey among a group of 325 teens to learn more about their habits and preferences regarding learning tools. We worked in partnership with two schools located in the city of Oceanside, in San Diego County, California. Students enrolled in grades 6,7 and 8 were surveyed and in a second educational institution we surveyed students in grades $9,10,11$ and 12 . Respectively, the average age of the surveyed groups was 13.5 years for the first school and 16 years for the second. The data was processed and analyzed using SPSS software.

This initiative aims at determining the potential of specific educational tools in order to efficiently develop the teenager public as a museum audience. By analyzing our results through the constructivist lens, we are trying to provide avenues to the different questions addressed in this article and, ultimately, to offer new information to museums who wish to increase the presence of teenagers within their walls. The post survey analysis have brought to light many interesting findings about the relationships between teenagers, educational tools and museums. It is worth mentioning the following result which, by itself, justifies the actual study: $66.1 \%$ of the teenagers asserted that they would visit museums more often if they were provided with educational tools that suited them. 